\title{
P111 Arterial Stiffness and Unattended and Attended BP Values
}

\author{
Anna Paini*, Carlo Aggiusti, Fabio Bertacchini, Deborah Stassaldi, Sara Capellini, Enrico Agabiti Rosei, \\ Maria Lorenza Muiesan, Massimo Salvetti
}

ASST Spedali Civili di Brescia, University of Brescia, Italy

\section{ABSTRACT}

Measurement of "unattended" blood pressure (BP) may reduce or eliminate the "white coat effect". Despite the possible advantages of this approach for BP measurement, only few studies analysed the relationship between unattended BP and cardiovascular events or with hypertension mediated organ damage (HMOD). The aim of our study was to evaluate the relationship between "attended" or "unattended" BP values and carotid-femoral pulse wave velocity (PWV) in 285 subjects undergoing a visit and assessment of arterial stiffness at an ESH Excellence Centre. Unattended BP (measured with the patient alone in the room, with an oscillometric device programmed to perform 3 BP measurements, at 1 minute intervals, after 5 minutes) and attended BP were measured with the same device, on the same day of the measurement of PWV, in random order.

Results: Mean age was $63 \pm 13$ years, mean BMI $26 \pm 4$, 47\% were female, 76\% had hypertension (55\% treated). Systolic unattended BP was lower as compared to attended SBP $(124.4 \pm 14.3$ vs $130.9 \pm 16.1 \mathrm{mmHg})$. PWV was similarly correlated with attended and unattended SBP values ( $r=0.428$ and $r=0.404, p<0.0001$, respectively). No difference for the prediction of increased arterial stiffness was observed at receiver operator curves (ROC) analysis: attended SBP area under the curve (AUC) $0.665,95 \%$ CI $0.607-0.720$ vs unattended SBP: AUC $0.651,95 \%$ CI $0.593-0.706, p$ for the comparison $=n s)$.

Conclusion: Attended and unattended BP values are similarly correlated with PWV, the gold standard measure of arterial stiffness. These findings may provide further information on the clinical value of unattended BP.

(c) 2019 Association for Research into Arterial Structure and Physiology. Publishing services by Atlantis Press International B.V. This is an open access article distributed under the CC BY-NC 4.0 license (http://creativecommons.org/licenses/by-nc/4.0/). 